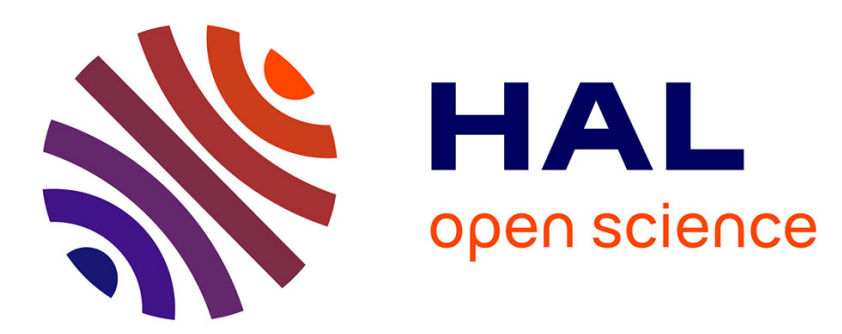

\title{
Algebraic estimation of a biased and noisy continuous signal via orthogonal polynomials
}

Rosane Ushirobira, Alban Quadrat

\section{To cite this version:}

Rosane Ushirobira, Alban Quadrat. Algebraic estimation of a biased and noisy continuous signal via orthogonal polynomials. CDC 2016 - 55th IEEE Conference on Decision and Control, Dec 2016, Las Vegas, United States. hal-01380320

\section{HAL Id: hal-01380320 \\ https://inria.hal.science/hal-01380320}

Submitted on 12 Oct 2016

HAL is a multi-disciplinary open access archive for the deposit and dissemination of scientific research documents, whether they are published or not. The documents may come from teaching and research institutions in France or abroad, or from public or private research centers.
L'archive ouverte pluridisciplinaire HAL, est destinée au dépôt et à la diffusion de documents scientifiques de niveau recherche, publiés ou non, émanant des établissements d'enseignement et de recherche français ou étrangers, des laboratoires publics ou privés. 


\title{
Algebraic estimation of a biased and noisy continuous signal via orthogonal polynomials
}

\author{
Rosane Ushirobira $^{1}$ and Alban Quadrat ${ }^{1}$
}

\begin{abstract}
Many important problems in Signal Processing and Control Engineering concern the reconstitution of a noisy biased signal. For this issue, in this paper we consider the signal written as an orthogonal polynomial series expansion and we provide an algebraic estimation of its coefficients. We specialize in Hermite polynomials. On the other hand, the dynamical system described by the noisy biased signal may be given by a differential equation associated with classical orthogonal polynomials. The signal may be recovered through the coefficients identification. As an example, we illustrate our algebraic method on the parameter estimation in the case of Hermite polynomials differential equations.
\end{abstract}

\section{INTRODUCTION}

Is is a widely known fact that parameter estimation is an important topic in various practical domains, such as in Control Engineering and Signal Processing. The list of applications concerning this question is extensively long.

Most traditional methods for solving this problem concern statistical approaches. From the last 10 years, an algebraic framework started to become more popular in the study of parameter identification. Algebraic approaches are mainly based on differential algebra concepts, operational calculus and module theory. An important paper in this subject is [3] where a closed loop parametric identification procedure for continuous-time constant linear systems is introduced. A very complete survey on algebraic identification can be found in [11]. Interesting applications within the algebraic context were provided in [7], [6], [10], [2], [9].

A longstanding essential problem in Signal Processing and Control Engineering consists in recovering a signal from a noisy biased measurement. One way to approach this question is to use a Taylor series expansion of the signal, or in a practical manner, to approximate the signal by a truncation of its Taylor series. It is in this way that numerical differentiation, i.e. the derivative estimation of the signal, has been the center of attention in countless papers. A worthto-mention work is the algebraic framework started with [8]. More details on this algebraic numerical differentiation technique can be found, for instance, in [4], [5], [6].

It seems of interest to study an alternative to numerical differentiation by considering the signal in another functions basis. A common signal decomposition, notably used in Signal Processing, originates from an orthogonal polynomial basis. In other words, the signal is written as an infinite sum of orthogonal polynomials with coefficients given by its respective basis projections which must then be identified.

\footnotetext{
${ }^{1}$ Rosane Ushirobira and Alban Quadrat are with Inria, Non-A team, 40 Avenue Halley, 59650 Villeneuve d'Ascq, France. rosane.ushirobiradinria.fr, alban.quadrateinria.fr.
}

Hence, the question of the reconstitution of the original signal fits in the category of parameter estimation issues.

In some problems, the dynamical system described by the signal $x$ can be defined by a second-order differential equation. In particular, the coefficients can be given by loworder polynomials. In this case, the identification of the ODE coefficients will allow the reconstitution of the noisy signal. Classical orthogonal polynomials, such as Jacobi, Legendre, Laguerre and Hermite polynomials, do satisfy such ODEs.

The algebraic method considered here is strongly based on Weyl algebra structural properties and one of its main advantages is to provide closed formulas for the parameter estimates. The reader may refer to works in [12], [13].

\section{GENERAL APPROACH}

In this section, we present the generic approaches aforementioned in the introduction. More precisely, the reconstitution of the signal via an orthogonal polynomials basis extension and the particular case of second-order timevarying systems are studied in this paper.

Throughout the text, $\mathbb{K}$ denotes a field of characteristic zero (e.g. $\mathbb{K}=\mathbb{R}$ or $\mathbb{C}$ ). To formalize our problem, we consider a signal $x$ that has to be recovered form a noisy biased signal $y$ defined by:

$$
y(t)=x(t)+\gamma+\varpi(t)
$$

where $\gamma$ is an unknown constant bias and $\varpi(t)$ is a zero-mean noise.

\section{A. Orthogonal basis}

The classical decomposition of a continuous signal $y$ in a basis of orthogonal polynomials $\mathcal{P}=\left\{P_{n}(t)\right\}_{n \geq 0}$ can be described by

$$
y(t)=\sum_{n \geq 0} \lambda_{n} P_{n}(t),
$$

where $\lambda_{n} \in \mathbb{R}$ corresponds to the projection of $y$ onto the orthogonal basis $\mathcal{P}$. Remark that $P_{n}$ might depend of unknown parameters, such as the Jacobi polynomials $P_{n}^{(\alpha, \beta)}$ (see Appendix).

Similar to the case of the Taylor expansion [8], an approximation of $y$ will be given by truncating the above series

$$
y(t) \approx y_{N}(t)=\sum_{n=0}^{N} \lambda_{n} P_{n}(t),
$$

for some $N>0$. We wish to identify the coefficients $\lambda_{0}, \ldots$, $\lambda_{N}$. The algebraic method proposed in this paper involves computations in the operational domain, so we apply the 
Laplace transform $\mathcal{L}$. First, recall that the action of $\mathcal{L}$ on a continuous function $f$ is given by

$$
\mathcal{L}(f)(s)=\int_{0}^{+\infty} e^{-s t} f(t) d t
$$

where $s$ denotes the Laplace variable. Applying $\mathcal{L}$ on (1) yields

$$
Y_{N}(s)=\sum_{n=0}^{N} \lambda_{n} \mathcal{L}\left(P_{n}\right)(s)
$$

where $Y_{N}$ denotes the Laplace transform of $y_{N}$. Then the idea is to individually estimate $\lambda_{i}, i=0, \ldots, N$. Our algebraic method proposes the elimination of all other coefficients except the one to be estimated. This elimination is realized through the action of differential operators called annihilators. After the elimination, the resulting equation in the time domain obtained with the action of the inverse Laplace transform provides closed formulas for estimating the $\lambda_{i}$.

\section{B. Second-order time-varying systems}

Now, we consider that the signal $x$ satisfies the following differential equation

$$
A(t) \ddot{x}(t)+B(t) \dot{x}(t)+C(t) x(t)=0,
$$

where $A, B$ and $C$ are elements of the polynomial ring $\mathbb{K}[t]$ in $t$ with coefficients in $\mathbb{K}$. Consider a signal $z$ by setting

$$
z(t):=x(t)+\gamma
$$

then using (2), $z$ satisfies:

$$
A(t) \ddot{z}(t)+B(t) \dot{z}(t)+C(t) z(t)-C(t) \gamma=0 .
$$

Our goal is to identify parameters appearing as the unknown coefficients of $A, B$ and $C$ in (3), since the bias will not be estimated. As before, we will then compute the Laplace transform of (3).

Prior to applying $\mathcal{L}$ to the above equation, few properties of the Laplace transform $\mathcal{L}$ of a signal $f$ are reviewed in the proposition below. We use the notation $\partial_{s}:=\frac{d}{d s}$.

Proposition 1:

1) $\mathcal{L}\left(f^{(n)}\right)(s)=s^{n} \mathcal{L}(f)(s)-\sum_{i=0}^{n-1} s^{n-i-1} f^{(i)}(0)$.

2) $\mathcal{L}\left(t^{n} \cdot f\right)(s)=(-1)^{n} \partial_{s}^{n}(\mathcal{L}(f)(s))$.

3) $\mathcal{L}(\gamma)=\frac{\gamma}{s}$, for all $\gamma \in \mathbb{K}$.

An immediate corollary can be derived from the above proposition.

Corollary 2: Let $R(t)=\sum_{k=0}^{n} a_{k} t^{k} \in \mathbb{K}[t]$ and $f \in \mathrm{C}^{\infty}\left(\mathbb{R}_{+}\right)$. Then, we have:

$\mathcal{L}(R . f)(s)=R\left(-\partial_{s}\right)(\mathcal{L}(f)(s))=\sum_{k=0}^{n}(-1)^{k} a_{k} \partial_{s}^{k}(\mathcal{L}(f)(s))$.

Hence, taking the Laplace transform of (3) and using the above properties, we obtain

$$
\begin{array}{r}
A\left(-\partial_{s}\right)\left(s^{2} Z(s)-s z(0)-\dot{z}(0)\right)+B\left(-\partial_{s}\right)(s Z(s)-z(0)) \\
+C\left(-\partial_{s}\right) Z(s)-C\left(-\partial_{s}\right) \frac{\gamma}{s}=0
\end{array}
$$

where $Z$ denotes the Laplace transform $\mathcal{L}(z)$ of $z$. Using the following notation

$$
\left\{\begin{array}{l}
\theta_{1}:=-x(0)=-z(0)+\gamma \\
\theta_{2}:=-\dot{x}(0)=-\dot{z}(0) \\
\theta_{3}:=-\gamma
\end{array}\right.
$$

and thus $-z(0)=\theta_{1}+\theta_{3}$, we obtain:

$$
\begin{aligned}
& \left(A\left(-\partial_{s}\right) s^{2}+B\left(-\partial_{s}\right) s+C\left(-\partial_{s}\right)\right) Z(s) \\
& +\left(A\left(-\partial_{s}\right) s+B\left(-\partial_{s}\right)\right)\left(\theta_{1}+\theta_{3}\right) \\
& +A\left(-\partial_{s}\right) \theta_{2}+C\left(-\partial_{s}\right) \frac{\theta_{3}}{s}=0
\end{aligned}
$$

In this paper, we shall only consider the following case

$$
\left\{\begin{array}{l}
A(t)=a_{2} t^{2}+a_{1} t+a_{0}, \\
B(t)=b_{1} t+b_{0} \\
C(t)=c
\end{array}\right.
$$

where $a_{2}, a_{1}, a_{0}, b_{1}, b_{0}, c \in \mathbb{K}$. Replacing these expressions in (4) yields

$$
\begin{gathered}
\widetilde{A}(s) \frac{d^{2}}{d s^{2}} Z(s)+\widetilde{B}(s) \frac{d}{d s} Z(s)+\widetilde{C}(s) Z(s) \\
+T_{1}(s) \theta_{1}+T_{2}(s) \theta_{2}+T_{3}(s) \theta_{3}=0
\end{gathered}
$$

where $\left\{\begin{array}{l}\widetilde{A}(s)=a_{2} s^{3}, \\ \widetilde{B}(s)=s^{2}\left(-s a_{1}+\left(4 a_{2}-b_{1}\right)\right), \\ \widetilde{C}(s)=s\left(a_{0} s^{2}+\left(-2 a_{1}+b_{0}\right) s+\left(2 a_{2}-b_{1}+c\right)\right), \\ T_{1}(s)=a_{0} s^{2}+\left(-a_{1}+b_{0}\right) s, \\ T_{2}(s)=a_{0} s \\ T_{3}(s)=T_{1}(s)+c .\end{array}\right.$

To estimate the coefficients of polynomials $A, B$ and $C$, algebraic manipulations are necessary to eliminate the undesired terms in (5). For that, algebraic operators called annihilators are then applied. The resulting expressions will contain only terms that are sought, allowing their identification in the time domain. To return to the time domain, the inverse Laplace transform $\mathcal{L}^{-1}$ is then applied. Recall that the inverse Laplace transform is given by

$$
\mathcal{L}^{-1}\left(\frac{1}{s^{m}} \frac{d^{p} Z(s)}{d s^{p}}\right)=\frac{(-1)^{p}}{(m-1) !} \int_{0}^{t} v_{m-1, p}(\tau) z(\tau) d \tau,
$$

with $v_{m, p}(\tau)=(t-\tau)^{m} \tau^{p}$, for all $p, m \in \mathbb{N}, m \geq 1$.

Let us consider classical examples.

Example 1: 1) Assume that $x(t)=P_{n}^{\alpha, \beta}(t)$, the $n$-th Jacobi polynomial depending on parameters $\alpha$ and $\beta$. In this case, the polynomials $A, B$ and $C$ are given by:

$$
\left\{\begin{array}{l}
A(t)=-t^{2}+1 \\
B(t)=-(\alpha+\beta+2) t+\beta-\alpha, \\
C(t)=n(n+\alpha+\beta+1) .
\end{array}\right.
$$


Then using (6), we have (5), where:

$$
\left\{\begin{array}{l}
\widetilde{A}(s)=-s^{3}, \\
\widetilde{B}(s)=s^{2}(\alpha+\beta-2), \\
\left.\widetilde{C}(s)=s\left(s^{2}+(\beta-\alpha)\right) s+(n+1)(n+\alpha+\beta)\right), \\
T_{1}(s)=s^{2}+(\beta-\alpha) s, \\
T_{2}(s)=s \\
T_{3}(s)=s^{2}+(\beta-\alpha) s+n(n+\alpha+\beta+1) .
\end{array}\right.
$$

2) Assume that $x(t)=P_{n}(t)$, the $n$-th Legendre which is a particular case of the $n$-th Jacobi polynomial for $\alpha=$ $\beta=0$. In this case, the $A, B$ and $C$ are given by:

$$
A(t)=-t^{2}+1, \quad B(t)=-2 t, \quad C(t)=n(n+1) .
$$

Then using (6), we have (5), where:

$$
\left\{\begin{array}{l}
\widetilde{A}(s)=-s^{3} \\
\widetilde{B}(s)=-2 s^{2} \\
\widetilde{C}(s)=s\left(s^{2}+n(n+1)\right) \\
T_{1}(s)=s^{2} \\
T_{2}(s)=s \\
T_{3}(s)=s^{2}+n(n+1)
\end{array}\right.
$$

3) Assume that $x(t)=L_{n}^{(\alpha)}(t)$, the $n$-th Laguerre polynomial depending on the parameter $\alpha$. Polynomials $A, B$ and $C$ in (3) are given by:

$$
A(t)=t, \quad B(t)=-t+\alpha+1, \quad C(t)=n .
$$

Then using [6], we have [5], where:

$$
\left\{\begin{array}{l}
\widetilde{A}(s)=0 \\
\widetilde{B}(s)=s^{2}(-s+1) \\
\widetilde{C}(s)=s((\alpha-1) s+(n+1)), \\
T_{1}(s)=\alpha s \\
T_{2}(s)=0 \\
T_{3}(s)=\alpha s+n
\end{array}\right.
$$

4) Assume that $x(t)=H_{n}(t)$, the $n$-th Hermite polynomial. Polynomials $A, B$ and $C$ in (3) are given by:

$$
A(t)=1, \quad B(t)=-2 t, \quad C(t)=2 n .
$$

Then, using (6), we have (5), where:

$$
\left\{\begin{array}{l}
\widetilde{A}(s)=0 \\
\widetilde{B}(s)=2 s^{2} \\
\widetilde{C}(s)=s\left(s^{2}+2(n+1)\right) \\
T_{1}(s)=s^{2} \\
T_{2}(s)=s \\
T_{3}(s)=s^{2}+2 n
\end{array}\right.
$$

\section{Motivational EXAMPLES}

\section{A. Hermite polynomials}

In this work, to illustrate one application of our algebraic estimation method, we will be particularly concerned with the Hermite polynomial series expansion of a continuous signal $x$. Hermite polynomials $H_{n}$ form an orthogonal set for $t \in \mathbb{R}$ with respect to the weight function $\mathrm{e}^{-t^{2}}$ (see Appendix). So any continuous function $y$ can be written as

$$
y(t)=\sum_{n \geq 0} \lambda_{n} H_{n}(t),
$$

where:

$$
\lambda_{n}=\frac{1}{2^{n} n ! \sqrt{\pi}} \int_{-\infty}^{\infty} y(\tau) H_{n}(\tau) \mathrm{e}^{-\tau^{2}} d \tau .
$$

An approximation of the function $y$ is provided by selecting a constant $N>0$ such that:

$$
y(t) \approx \sum_{n=0}^{N} \lambda_{n} H_{n}(t) .
$$

We denote this polynomial approximation by $y_{N}(t)$.

The aim is to estimate the terms $\lambda_{n}$, for $n=0, \ldots, N$. Notice that $y$ represents the measured signal from a signal $x$ with some negligible noise, hence we may consider only $y$. As we mentioned in the previous subsection, the first step is to apply the Laplace transform on (12):

$$
Y_{N}(s)=\sum_{n=0}^{N} \lambda_{n} \mathcal{L}\left(H_{n}\right)(s),
$$

where $Y_{N}$ denotes the Laplace transform of $y_{N}$. From the definition of Hermite polynomials (see Appendix), it follows

$$
H_{n}(t)=2^{n} t^{n}+h_{n}(t)=2^{n} t^{n}+\eta_{n, n-2} t^{n-2}+\cdots+\eta_{n, m} t^{m}
$$

with $m=n \bmod 2$ (i.e., $m=0$ if $n$ is even and $m=1$ if $n$ is odd). So, denoting $n=2 j$ or $n=2 j+1$, it results that

$$
P_{n}(s):=\mathcal{L}\left(H_{n}\right)(s)=2^{n} \frac{n !}{s^{n+1}}+\sum_{k=1}^{j} \eta_{n, n-2 k} \frac{(n-2 k) !}{s^{n-2 k+1}} .
$$

Multiplying (13) by the highest power of $s$ to eliminate denominators gives:

$s^{N+1} Y_{N}(s)=\lambda_{N}\left(2^{N} N !+\sum_{k=1}^{j} \eta_{N, N-2 k} \frac{(N-2 k) !}{s^{N-2 k+1}}\right)+\sum_{n=0}^{N-1} \lambda_{n} P_{n}(s)$.

The parameters $\lambda_{i}$ up to order $N$ will be estimated individually. Denote the set of parameters to be estimated by:

$$
\Theta=\left\{\lambda_{1}, \ldots, \lambda_{N}\right\} .
$$

We start with the dominant coefficient $\lambda_{N}$ and use the notation $\Theta_{\text {est }}=\left\{\lambda_{N}\right\}$. Then, accordingly separate the terms in (14) to rewrite the equation into the following relation:

$$
P\left(s, \partial_{s}\right) Y_{N}(s)+Q(s)+\bar{Q}(s)=0,
$$

where $P$ is a differential operator on the Laplace variable $s$ with coefficients in the field $\mathbb{R}_{\Theta}:=\mathbb{R}(\Theta)$ (i.e. an algebraic extension of $\mathbb{R}$ containing the set $\Theta$ ), so $P \in \mathbb{R}_{\Theta}\left[s, \partial_{s}\right], Q$ is 
a polynomial in $s$ with coefficients in $\mathbb{R}_{\Theta_{\mathrm{est}}}:=\mathbb{R}\left(\Theta_{\mathrm{est}}\right)$, and $\bar{Q}$ is a polynomial in $s$ with coefficients in $\mathbb{R}_{\Theta}$ :

$$
\left\{\begin{array}{l}
P(s)=s^{N+1} \partial_{s}+\left(s^{3}+(2 n+2) s\right), \\
Q(s)=\lambda_{N} P_{N}(s), \\
\bar{Q}(s)=\sum_{n=0}^{N-1} \lambda_{n} P_{n}(s) .
\end{array}\right.
$$

To determine a closed formula for the estimation of $\lambda_{N}$, the polynomial $\bar{Q}$ must be eliminated from (15). This elimination is realized by the action of differential operators, providing an expression containing only $\lambda_{N}, Y_{N}$ and its derivatives. A time-domain expression for $\lambda_{N}$ can then be obtained by applying the inverse Laplace transform. The same procedure is applied to estimate all remaining $\lambda_{i}$.

For instance, if $M=3$, then we obtain from (14) that

$s^{4} Y_{N}(s)=-\left(12 s^{2}-48\right) \lambda_{3}-\left(2 s^{3}-8 s\right) \lambda_{2}+s^{3} \lambda_{0}+2 s^{2} \lambda_{1}$.

Starting with $\Theta_{\text {est }}=\left\{\lambda_{3}\right\}$, the relation obtained is:

$$
\begin{aligned}
& P(s)=s^{4}, Q(s)=-\left(12 s^{2}-48\right) \lambda_{3}, \\
& \bar{Q}(s)=-\left(2 s^{3}-8 s\right) \lambda_{2}+s^{3} \lambda_{0}+2 s^{2} \lambda_{1} .
\end{aligned}
$$

\section{B. Second-order time-varying systems}

Particular cases of second-order time-varying system are described by differential equations originated by orthogonal polynomials as seen in Section II-B.

Let $\Theta$ be the set of all parameters in the operational equations (8), (9), (10), (11). Since we do not wish to identify the bias, the parameters to be estimated are then $\theta_{1}$ and $\theta_{2}$ and sometimes $\alpha$ and/or $\beta$.

In the next section, to illustrate our algebraic method for parameter estimation, we will focus on Example 4 and identify parameters in the case of Hermite polynomials.

After the passage from the time domain to the operational domain via the Laplace transform, the next step in the estimation procedure consists in rewriting (11) according to the parameters to be identified. For instance, if we wish to estimate $\theta_{1}$ and $\theta_{2}$, we define a set $\Theta_{\text {est }}$ by:

$$
\Theta_{\mathrm{est}}=\left\{\theta_{1}, \theta_{2}\right\} .
$$

So $\Theta_{\text {est }} \subset \Theta$. From (11) we can write the relation:

$$
P\left(s, \partial_{s}\right) Z(s)+Q(s)+\bar{Q}(s)=0
$$

where

$$
\begin{aligned}
P\left(s, \partial_{s}\right) & =2 s^{2} \partial_{s}+\left(s^{3}+(2 n+2) s\right) \\
Q(s) & =s^{2} \theta_{1}+s \theta_{2} \\
\bar{Q}(s) & =\left(s^{2}+2 n\right) \theta_{3}
\end{aligned}
$$

Annihilators are then applied on the relation $\mathcal{R}$ to eliminate $\bar{Q}$. The remaining terms provide a system of equations in $\Theta_{\text {est }}$. A short description on the algebraic framework used to design annihilators can be found in the next section.

\section{ANNIHILATORS}

Algebraic concepts and some structural properties can be found in the Appendix VII-B.

For the sake of simplicity, from now on we consider $\mathbb{K}=$ $\mathbb{R}$. We also set $\mathrm{B}:=\mathrm{B}_{1}(\mathbb{R})=\mathbb{R}(s)\left[\partial_{s}\right]$.

Definition 1: Let $R \in \mathbb{R}_{\Theta}[s]$. A R-annihilator w.r.t. $\mathrm{B}$ is an element of $\mathrm{Ann}_{\mathrm{B}}(R)=\{F \in \mathrm{B} \mid F(R)=0\}$.

By Proposition 9. Appendix VII-B B is a left principal domain. Therefore then $\operatorname{Ann}_{\mathrm{B}}(R)$ is a left principal ideal (i.e. it is generated by a unique $\Pi_{\min } \in \mathrm{B}$, up to multiplication by a polynomial in $\mathrm{B}$ ). That means $\operatorname{Ann}_{\mathrm{B}}(R)=\mathrm{B} \Pi_{\text {min }}$. We call $\Pi_{\text {min }}$ a minimal $Q$-annihilator w.r.t. B. Remark that $\operatorname{Ann}_{\mathrm{B}}(R)$ contains annihilators in finite integral form, i.e. operators with coefficients in $\mathbb{R}\left[\frac{1}{s}\right]$. The following lemmas are useful:

Lemma 3: Consider $R(s)=s^{n}, n \in \mathbb{N}$. A minimal $R$ annihilator is given by $\Pi_{n}=s \partial_{s}-n$.

For $m, n \in \mathbb{N}$, the operators $\Pi_{m}$ and $\Pi_{n}$ commute. Thus, one has the following Lemma

Lemma 4: Let $P_{1}, P_{2} \in \mathbb{R}_{\Theta}[s]$. Let $F_{i}$ be a $P_{i}$-annihilator $(i=1,2)$ such that $F_{1} F_{2}=F_{2} F_{1}$. Then $F_{1} F_{2}$ is a $\left(\mu P_{1}+\eta P_{2}\right)$ annihilator for all $\mu, \eta \in \mathbb{R}_{\Theta}$.

Lemma 5: Let $R \in \mathbb{R}_{\Theta}[s]$. Then a minimal $R$-annihilator w.r.t $\mathrm{B}_{\Theta_{\mathrm{est}}}$ is $\Pi_{\min }=R \partial_{s}-\partial_{s}(R)$.

It may happen that a $\bar{Q}$-annihilator eliminates all terms in the relation $\mathcal{R}$ that contain the parameters to be estimated. Hence, another important concept lies in the definition of an estimator.

Definition 2: An estimator $\Pi \in \mathrm{B}$ a $\bar{Q}$-annihilator satisfying coeffs $\left(\Pi((\mathcal{R})) \cap \mathbb{R}_{\Theta_{\text {est }}}=\emptyset\right.$..

Now, from 17] we have:

$$
\bar{Q}(s)=\left(s^{2}+2 n\right) \theta_{3} .
$$

Since the degree of $\bar{Q}$ in $s$ is equal to 2 , then $\Pi=\partial_{s}^{3}$ is a $\bar{Q}$-annihilator. But the action of $\Pi$ on the relation $\mathcal{R}(17)$ also eliminates the polynomial $Q$ that contains the parameters to be identified. So $\Pi$ is not a estimator.

Lemma 3 gives a minimal $\bar{Q}$-annihilator for $s^{2}$ :

$$
\pi_{1}=s \partial_{s}-2 \text {. }
$$

To complete annihilate $\bar{Q}$, it is enough to apply $\pi_{2}=\partial_{s}$ on $\pi_{1}(\bar{Q})$. That gives a $\bar{Q}$-annihilator:

$$
\Pi=\pi_{2} \pi_{1}=s \partial_{s}^{2}-\partial_{s} .
$$

Moreover, Lemma 5 provides a minimal $\bar{Q}$-annihilator w.r.t. B:

$$
\Phi=\left(s^{2}+2 n\right) \partial_{s}-2 s .
$$

\section{EXAMPLES}

\section{Example 1: Hermite expansion series of $x$}

In this subsection, we work with a truncate series expansion of order 3 and illustrate our method with 16 by giving the estimation of $\lambda_{3}$.

From (16), we have:

$$
\begin{aligned}
& P(s)=s^{4}, Q(s)=-\left(12 s^{2}-48\right) \lambda_{3}, \\
& \bar{Q}(s)=-\left(2 s^{3}-8 s\right) \lambda_{2}+s^{3} \lambda_{0}+2 s^{2} \lambda_{1} .
\end{aligned}
$$


To annihilate $\bar{Q}$, we begin by eliminating $\lambda_{0}$. From Lemma 3 . we apply $\pi_{1}=s \partial_{s}-3$ on $\bar{Q}(16)$ and obtain $\pi_{1}(\bar{Q})=2 s^{2} \lambda_{1}+$ $16 \lambda_{2} s$. Using the same Lemma twice, we apply subsequently $\pi_{2}=s \partial_{s}-2$ and $\pi_{3}=s \partial_{s}-1$ to completely annihilate $\bar{Q}$.

The annihilator $\pi=\pi_{3} \pi_{2} \pi_{1}$ can be rewritten in the canonical form, using Example 2 .

$$
\pi=s^{3} \partial_{s}{ }^{3}-3 s^{2} \partial_{s}^{2}+6 \partial_{s} s-6 .
$$

We apply $\pi$ on 16 and it follows:

$6 s^{4} Y(s)+288 a_{3}+18 s^{5} \frac{\mathrm{d}}{\mathrm{d} s} Y(s)+9 s^{6} \frac{\mathrm{d}^{2}}{\mathrm{~d} s^{2}} Y(s)+s^{7} \frac{\mathrm{d}^{3}}{\mathrm{~d} s^{3}} X(s)=0$.

The inverse Laplace transform (7) helps to return to the time domain:

$$
\begin{aligned}
& \int_{0}^{t} v_{3,0}(\tau) y(\tau) d \tau+\frac{2 a_{3} t^{7}}{35} \\
& +\int_{0}^{t}\left(-9 v_{2,1}(\tau)+9 v_{1,2}(\tau)-v_{0,3}(\tau)\right) y(\tau) d \tau=0
\end{aligned}
$$

Finally, solving (18) and changing variables:

$$
\lambda_{3}=\frac{35 \int_{0}^{1} y(t v)\left(20 v^{3}-30 v^{2}+12 v-1\right) d v}{2 t^{3}}
$$

\section{Example 2: Hermite polynomial}

Here, we consider a second-order time-varying system described by the following differential equation:

$$
\ddot{z}(t)-2 t \dot{z}(t)+2 n z(t)-2 n \gamma=0
$$

As seen in (11), Section II, the above equation yields in the operational domain:

$$
\begin{aligned}
& 2 s^{2} \frac{\mathrm{d}}{\mathrm{d} s} Z(s)+\left(s^{3}+2(n+1) s\right) Z(s)+s^{2} \theta_{1}+s \theta_{2} \\
& +\left(s^{2}+2 n\right) \theta_{3}=0 .
\end{aligned}
$$

The aim is to identify the parameter $n$ in (19). So the relation $\mathcal{R}$ that will be considered is:

$$
P\left(s, \partial_{s}\right) Z(s)+Q(s)+\bar{Q}(s)=0 .
$$

with $P\left(s, \partial_{s}\right)=2 s^{2} \partial_{s}+\left(s^{3}+(2 n+2) s\right), Q(s)=0$ and $\bar{Q}(s)=+s^{2} \theta_{1}+s \theta_{2}+\left(s^{2}+2 n\right) \theta_{3}$. Using Lemma 3 three times, a $\bar{Q}$-annihilator in the canonical form is given by:

$$
\pi=s \partial_{s}^{3} .
$$

and provides in the operational domain:

$$
\begin{aligned}
& \left(2 s^{2} \frac{\mathrm{d}^{3}}{\mathrm{~d} s^{3}} Z(s)+6 s \frac{\mathrm{d}^{2}}{\mathrm{~d} s^{2}} Z(s)\right) n+18 s^{2} \frac{\mathrm{d}}{\mathrm{d} s} Z(s) \\
& +\left(9 s^{3}+18 s\right) \frac{\mathrm{d}^{2}}{\mathrm{~d} s^{2}} Z(s)+\left(s^{4}+14 s^{2}\right) \frac{\mathrm{d}^{3}}{\mathrm{~d} s^{3}} Z(s) \\
& +2 s^{3} \frac{\mathrm{d}^{4}}{\mathrm{~d} s^{4}} Z(s)+6 s Z(s)=0 .
\end{aligned}
$$

The equation allowing the identification of $n$ is provided by:

$$
n=\frac{\mathcal{A}}{\mathcal{B}}
$$

$$
\begin{aligned}
\mathcal{A}= & \int_{0}^{t}\left(-3 t^{3} \tau^{2}+16 t^{2} \tau^{3}-25 t \tau^{4}+12 \tau^{5}-t^{3}+12 t^{2} \tau-30 \tau^{2} t\right. \\
& \left.+20 \tau^{3}\right) z(\tau) d \tau \\
\mathcal{B}= & \int_{0}^{t}\left(t^{3} \tau^{2}-4 t^{2} \tau^{3}+5 t \tau^{4}-2 \tau^{5}\right) z(\tau) d \tau
\end{aligned}
$$

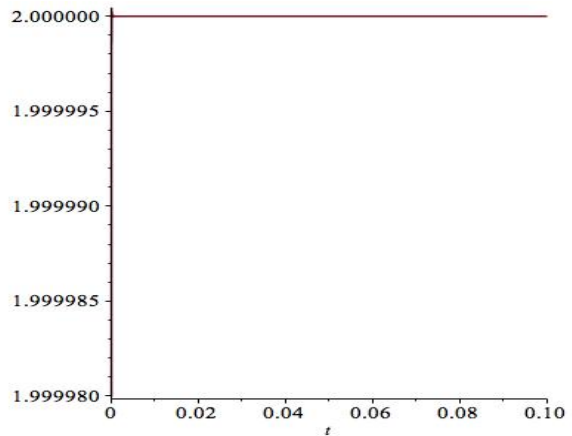

Fig. 1. The result of the identification of $n$

In the case of the second-order Hermite Polynomial $H_{2}(t)$, we consider $z(t)=H_{2}(t)+\varpi(t)$ with $\varpi(t)$ some noise. Fig. 1 shows the simulation using the above identification of $n$ for $n=2$.

\section{CONCLUSION}

In this paper, we addressed the problem of the reconstitution of a noisy biased signal that is involved in many important problems in Signal Processing and Control Engineering. Two approaches were studied for this issue. One of them was the use of an orthogonal polynomial series expansion for the signal. In this approach, thanks to an algebraic framework we provided an estimation of its coefficients in the particular case of Hermite polynomials. This choice was motivated by the common use of these polynomials in the domain of Signal Processing. Future work will include a broader study of other classical polynomials, such as Jacobi, Legendre and Laguerre. Errors arising from truncating the series expansion should be also analyzed.

The second approach presented in this paper concerns the case where the dynamical system described by the noisy biased signal is given by a differential equation satisfied by classical orthogonal polynomials. The ODE coefficients identification allowed the signal to be recovered. An example for second-degree Hermite polynomial was chosen to illustrate our algebraic methods. Further research will focus on different choices of parameters to be estimated, as well as on different orders for the ODE polynomial coefficients.

It should be stressed that the choice of differential operators called annihilators is a crucial step in the algebraic procedure allowing a better posed problem and consequently, better estimates.

\section{APPENDIX}

\section{A. Classical orthogonal polynomials}

Here, we recall the definition of some classical orthogonal polynomials. In particular, properties of Jacobi and Hermite polynomials are provided. For more details, we refer to [1].

1) Jacobi polynomials: 
Jacobi polynomials can be defined by Rodrigues' formula:

$$
\begin{aligned}
P_{n}^{(\alpha, \beta)}(z)=\frac{(-1)^{n}}{2^{n} n !}(1-z)^{-\alpha}(1+z)^{-\beta} . & \frac{d^{n}}{d z^{n}}\left((1-z)^{\alpha}(1+z)^{\beta}\left(1-z^{2}\right)^{n}\right) .
\end{aligned}
$$

2) Hermite polynomials: The definition of Hermite polynomials is given by:

$$
H_{n}(t)=(-1)^{n} \mathrm{e}^{t^{2}} \frac{d^{n}}{d t^{n}} \mathrm{e}^{-t^{2}} .
$$

It follows that Hermite polynomials of even degree are even functions and polynomials of odd degree are odd functions:

$$
H_{n}(t)=2^{n} t^{n}+h_{n}(t)
$$

where $h_{n}(t)$ is a polynomial with non-zero coefficients for all even powers of $t$ smaller than $n$ if $n$ is even and for all odd powers if $n$ is odd. Hermite polynomials are orthogonal with respect to the scalar product defined by the weight function $w(t)=\mathrm{e}^{-t^{2}}:$

$$
\left\langle H_{n}(t), H_{m}(t)\right\rangle=\int_{-\infty}^{\infty} H_{m}(\tau) H_{n}(\tau) w(\tau) d \tau=0, \quad m \neq n .
$$

\section{B. The Weyl Algebra: basic notions}

Definition 3: Let $\mathbb{K}$ be a field of characteristic zero. Let $k \in \mathbb{N} \backslash\{0\}$. The Weyl algebra $\mathrm{A}_{k}=\mathrm{A}_{k}(\mathbb{K})$ is the free $\mathbb{K}$ algebra generated by $p_{1}, q_{1}, \ldots, p_{k}, q_{k}$ satisfying the relations

$$
1 \leq i, j \leq k, \quad\left[p_{i}, q_{j}\right]=\delta_{i j}, \quad\left[p_{i}, p_{j}\right]=\left[q_{i}, q_{j}\right]=0,
$$

where $[\cdot, \cdot]$ is the commutator defined by $[u, v]:=u v-v u$ for all $u, v \in \mathrm{A}_{k}(\mathbb{K})$ and $\delta_{i j}$ is the Kronecker function, i.e. $\delta_{i j}=1$, if $i=j$ and 0 , if $i \neq j$.

A useful realization of the Weyl algebra $A_{k}$ is to consider it as the $\mathbb{K}$-algebra of polynomial differential operators on $\mathbb{K}\left[s_{1}, \ldots, s_{k}\right]$ such that $p_{i}:=\partial_{s_{i}}=\frac{\partial}{\partial s_{i}}$ is the derivative with respect to $s_{i}$ and $q_{i}:=s_{i}$ is interpreted as the multiplication operator $p\left(s_{1}, \ldots, s_{k}\right) \longmapsto s_{i} p\left(s_{1}, \ldots, s_{k}\right)$, for $1 \leq i \leq k$.

As a consequence, we can write:

$$
\mathrm{A}_{k}=\mathbb{K}\left[q_{1}, \ldots, q_{k}\right]\left[p_{1}, \ldots, p_{k}\right]=\mathbb{K}\left[s_{1}, \ldots, s_{k}\right]\left[\partial_{s_{1}}, \ldots, \partial_{s_{k}}\right] .
$$

Remark 6: The same notation is used for the variable $s_{i}$ and for the operator "multiplication by $s_{i}$ ".

A closely related algebra to $A_{k}(\mathbb{K})$ is defined as the differential operators on $\mathbb{K}\left[s_{1}, \ldots, s_{k}\right]$ with coefficients in the rational functions field $\mathbb{K}\left(s_{1}, \ldots, s_{k}\right)$. We denote it by $\mathrm{B}_{k}(\mathbb{K})$, or $\mathrm{B}_{k}$ for short. We can write:

$$
\mathrm{B}_{k}:=\mathbb{K}\left(q_{1}, \ldots, q_{k}\right)\left[p_{1}, \ldots, p_{k}\right]=\mathbb{K}\left(s_{1}, \ldots, s_{k}\right)\left[\partial_{s_{1}}, \ldots, \partial_{s_{k}}\right] .
$$

Proposition 7: A basis for $\mathrm{A}_{k}$ is given by $\left\{q^{I} p^{J} \mid I, J \in \mathbb{N}^{k}\right\}$ where $q^{I}:=q_{1}^{i_{1}} \ldots q_{k}^{i_{k}}$ and $p^{J}:=p_{1}^{j_{1}} \ldots p_{k}^{j_{k}}$ if $I=\left(i_{1}, \ldots, i_{k}\right)$ and $J=\left(j_{1}, \ldots, j_{k}\right)$. So an operator $F \in \mathrm{A}_{k}$ can be written in a canonical form,

$$
F=\sum_{I, J} \lambda_{I J} q^{I} p^{J} \quad \text { with } \quad \lambda_{I J} \in \mathbb{K}
$$

Example 2: We need later the fallowing useful identity:

$$
p^{n} q^{m}=q^{m} p^{n}+\sum_{k=1}^{n}\left(\begin{array}{c}
n \\
i
\end{array}\right)\left(\begin{array}{c}
m \\
i
\end{array}\right) i ! q^{m-i} p^{n-i}
$$

An element $F \in \mathrm{B}_{k}$ can be similarly written as

$$
F=\sum_{I} \lambda_{I} g_{I}(s) p^{I}, \text { where } g_{I}(s) \in \mathbb{K}\left(s_{1}, \ldots, s_{k}\right) .
$$

The order of an element $F \in \mathrm{B}_{k}, F=\sum_{I} \lambda_{I} g_{I}(s) p^{I}$ is defined as $\operatorname{ord}(F):=\max \left\{\left[I|| g_{I}(s) \neq 0\right\}\right.$. The same definition holds for the Weyl algebra $A_{k}$ since $A_{k} \subset B_{k}$. Some properties of $\mathrm{A}_{k}$ and $\mathrm{B}_{k}$ are given by the following propositions:

Proposition 8: $\mathrm{A}_{k}$ is a domain. Moreover, $\mathrm{A}_{k}$ is simple and Noetherian.

These properties are shared by $B_{k}$. Furthermore, $A_{k}$ is neither a principal right domain, nor a principal left domain, while this is true for $\mathrm{B}_{k}$ :

Proposition 9: $\mathrm{B}_{1}$ admits a left division algorithm, that is, if $F, G \in \mathrm{B}_{1}$, then there exists $Q, R \in \mathrm{B}_{1}$ such that $F=Q G+$ $R$ and $\operatorname{ord}(R)<\operatorname{ord}(G)$. So $\mathrm{B}_{1}$ is a principal left domain.

Lastly, it follows from the fact that $\frac{d}{d s}$ is a derivation:

Proposition 10 (Derivation): Given $P_{1}, P_{2} \in \mathbb{K}[s]$, we have (Leibniz rule):

$$
\frac{d^{n}}{d s^{n}}\left(\begin{array}{ll}
P_{1} & P_{2}
\end{array}\right)=\sum_{k=0}^{n}\left(\begin{array}{l}
n \\
k
\end{array}\right) \frac{d^{k} P_{1}}{d s^{k}} \frac{d^{n-k} P_{2}}{d s^{n-k}}
$$

\section{REFERENCES}

[1] M. Abramowitz. Handbook of Mathematical Functions, With Formulas, Graphs, and Mathematical Tables,. Dover Publications, Incorporated, 1974.

[2] J. Cortés-Romero, C. García-Rodríguez, A. Luviano-Juárez, and H. Sira-Ramírez. Algebraic parameter identification for induction motors. In IECON 2011 - 37th Annual Conference on IEEE Industrial Electronics Society, pages 1734-1740, Nov 2011.

[3] M. Fliess and H. Sira-Ramírez. An algebraic framework for linear identification. ESAIM Control Optim. Calc. Variat., 9:151-168, 2003.

[4] D.Y. Liu, O. Gibaru, and W. Perruquetti. Error analysis for a class of numerical differentiator: application to state observation. In 48th IEEE Conf. on Decision and Control, Shanghai, China, 2009.

[5] D.Y. Liu, O. Gibaru, W. Perruquetti, M. Fliess, and M. Mboup. An error analysis in the algebraic estimation of a noisy sinusoidal signal. In 16th Mediterranean Conf. on Control and Automation (MED'08), Ajaccio, France, 2008.

[6] M. Mboup. Parameter estimation for signals described by differential equations. Applicable Analysis, 88:29-52, 2009.

[7] M. Mboup. Neural spike detection and localisation via volterra filtering. In 22nd IEEE Workshop on Machine Learning for Signal Processing, Santander, Spain, September 2012.

[8] M. Mboup, C. Join, and M. Fliess. Numerical differentiation with annihilators in noisy environement. Numerical Algorithms, 50:439467, 2009.

[9] L. Menhour, B. d'Andrea Novel, C. Boussard, M. Fliess, and H. Mounier. Algebraic nonlinear estimation and flatness-based lateral/longitudinal control for automotive vehicles. In Intelligent Transportation Systems (ITSC), 2011 14th International IEEE Conference on, pages 463-468, Oct 2011.

[10] W. Perruquetti, P. Bonnet, M. Mboup, R. Ushirobira, and P. Fraisse. An algebraic approach for humane posture estimation in the sagital plane using accelerometer noisy signal. In Proc. 51st IEEE Conf. Decision Control, page 6, Dec. 2012.

[11] H Sira-Ramírez, C. G. Rodríguez, J. C. Romero, and A. L. Juárez. Algebraic Identification and Estimation Methods in Feedback Control Systems. Wiley Publishing, 1st edition, 2014.

[12] R. Ushirobira, W. Perruquetti, M. Mboup, and M. Fliess. Algebraic parameter estimation of a biased sinusoidal waveform signal from noisy data. In Sysid 2012, 16th IFAC Symposium on System Identification, Brussels, Belgique, 2012.

[13] R. Ushirobira, W. Perruquetti, M. Mboup, and M. Fliess. Algebraic parameter estimation of a multi-sinusoidal waveform signal from noisy data. In European Control Conference, Zurich, Suisse, April 2013. 